\section{PRACTICE PERSPECTIVES}

\author{
W. Brinjikji \\ D.F. Kallmes \\ G. Lanzino \\ H.J. Cloft
}

\title{
Hospitalization Costs for Endovascular and Surgical Treatment of Ruptured Aneurysms in the United States Are Substantially Higher Than Medicare Payments
}

BACKGROUND AND PURPOSE: Endovascular and surgical options are both available for treatment of ruptured cerebral aneurysms. Knowledge of the costs relative to Medicare reimbursement for hospitalization of these patients is important for understanding the economic impact of these patients on hospitals.

MATERIALS AND METHODS: Using the NIS, we identified hospitalizations for clipping and coiling of ruptured cerebral aneurysms from 2001 to 2008 by cross-matching ICD-9 codes for diagnosis of ruptured aneurysm with procedure codes for clipping or coiling of cerebral aneurysms. Hospital costs for 2008 were correlated with age, sex, and discharge status. For discharges of Medicare patients, we compared Medicare payments with costs for respective MS-DRG.

RESULTS: For 2008, the average Medicare payment for craniotomy and endovascular intracranial procedures without complication (MS-DRG 22) was $\$ 30,380$. Medicare discharges with DRG 22, for patients undergoing clipping had median costs of $\$ 59,799$ in 2008. Those undergoing coiling had median costs of $\$ 36,543$. Reimbursement for discharges with complications or comorbidities (MSDRG 21) was $\$ 36,304$. Median costs for hospitalization of clipped patients with MS DRG 21 was $\$ 79,916$ and for coiled patients, $\$ 56,910$. Reimbursement for MS-DRG 20 (major complications or comorbidities) was $\$ 41,748$, with patients undergoing clipping incurring a median cost of $\$ 83,737$ and those undergoing coiling incurring a median cost of $\$ 83,277$.

CONCLUSIONS: Hospitalization costs for patients undergoing clipping and coiling of unruptured cerebral aneurysms are substantially higher than Medicare payments.

ABBREVIATIONS: CM = Clinical Modification; DRG = diagnostic-related groups; ICD = International Classification of Diseases; IQR = interquartile range; MS-DRG = Medicare Severity Diagnostic-Related Groups; NIS = National Inpatient Sample

$\mathbf{T}$ he number of patients in the United States who undergo treatment for ruptured aneurysm with endovascular coiling has increased dramatically between 2000 and 2006. ${ }^{1}$ Given this recent change in the treatment of ruptured aneurysms, assessing the trends in hospitalization costs associated with treatment with coiling and clipping would be economically important. Hospitalization costs are important to understand because they may exceed Medicare payments. In this study, we used the NIS data base to assess the recent hospitalization costs associated with clipping and coiling of ruptured cerebral aneurysms.

\section{Materials and Methods}

\section{Patient Population}

We collected and analyzed data from the NIS hospital discharge data base for 2001-2008, which was acquired from the Healthcare Cost and Utilization Project of the Agency for Healthcare Research and Quality, Rockville, Maryland. The NIS is a hospital discharge data

\section{Received June 28, 2011; accepted after revision October 11}

From the Mayo Medical School (W.B.) and Departments of Radiology (D.F.K., G.L., H.J.C.) and Neurosurgery (D.F.K., G.L., H.J.C.), Mayo Clinic, Rochester, Minnesota.

Please address correspondence to Harry J. Cloft, MD, PhD, Mayo Clinic, 200 SW First St, Rochester, MN 55905; e-mail: cloft.harry@mayo.edu

Indicates article with supplemental on-line table.

http://dx.doi.org/10.3174/ajnr.A2938 base that represents $20 \%$ of all inpatient admissions to nonfederal hospitals in the United States. All discharges included in this study carried a primary diagnosis of subarachnoid hemorrhage (Code 430 in the ICD-9-CM and a primary ICD-9-CM procedure code of "clipping of aneurysm" (ICD-9-CM code 39.51) or of "coiling of aneurysm," which included "other repair of aneurysm"(ICD-9-CM code 39.52), "endovascular repair or occlusion of head and neck vessels" (ICD-9-CM code 39.72), and "other endovascular repair (of aneurysm) of other vessels” (ICD-9-CM code 39.79).

We examined the correlation between the following variables and total costs in 2008: age, sex, and discharge status (home/short-term facility, long-term facility, and in-hospital death). For 2001-2008, we calculated median hospital costs for hospitalization for clipping and coiling to evaluate cost trends with time. Cost data were obtained by multiplying the hospital charges by the group-average cost-to-charge ratio for each hospital in the data base. Group average cost-to-charge ratio and hospital charges were available in the NIS data base. All costs were converted to their dollar value in 2008 by using the consumer price index calculator available at http://data.bls.gov/cgi-bin/ cpicalc.pl. Data on mean costs are provided in the On-line Table.

\section{Comparison with Medicare Reimbursements}

Medicare payments are public information (http://www.cms.hhs. gov) and were compared with the 2008 hospitalization cost data. When comparing costs with Medicare reimbursement, we performed a separate analysis only including those patients whose hospitaliza- 


\begin{tabular}{|c|c|c|c|c|c|c|c|}
\hline \multicolumn{8}{|c|}{2008 hospitalization costs for clipping and coiling of ruptured intracranial aneurysms } \\
\hline \multirow[b]{2}{*}{ Variable } & \multicolumn{3}{|c|}{ Clipping } & \multicolumn{3}{|c|}{ Coiling } & \multirow[b]{2}{*}{$\begin{array}{c}P \text { Value Clipping } \\
\text { vs Coiling }\end{array}$} \\
\hline & $\begin{array}{l}\text { Median Cost } \\
(\$ 2008)\end{array}$ & IOR & $P$ Value & $\begin{array}{l}\text { Median Cost } \\
(\$ 2008)\end{array}$ & IOR & $P$ Value & \\
\hline All patients & 69,353 & $46,377-105,049$ & - & 66,290 & $42,214-110,302$ & - & .45 \\
\hline \multicolumn{8}{|l|}{ Sex } \\
\hline Male & 70,982 & $46,967-110,947$ & .27 & 66,931 & $43,927-112,337$ & .36 & .61 \\
\hline Female & 69,213 & $45,652-10,1249$ & & 65,805 & $41,344-107,759$ & & .62 \\
\hline \multicolumn{8}{|l|}{ Age } \\
\hline$<65$ & 65,751 & $44,087-97,560$ & $<.0001$ & 64,252 & $40,503-107,503$ & .01 & .89 \\
\hline$\geq 65$ & 90,745 & $58,390-12,3538$ & & 74,918 & $47,799-117,142$ & & .02 \\
\hline \multicolumn{8}{|c|}{ All patients by discharge status } \\
\hline Home & 56,201 & $39,584-79,938$ & $<.0001$ & 51,822 & $34,992-74,887$ & $<.0001$ & $<.0001$ \\
\hline Short-term facility & 92,340 & $59,431-128,177$ & & 85,192 & $47,144-122,864$ & & .0002 \\
\hline Long-term facility & 95,995 & $70,004-133,070$ & & 99,349 & $66,979-146,196$ & & .42 \\
\hline Dead & 57,268 & $29,774-155,180$ & & 56,699 & $39,071-89,639$ & & .91 \\
\hline \multicolumn{8}{|c|}{ Age $<65$ by discharge status } \\
\hline Home & 58,007 & $40,107-83,965$ & $<.0001$ & 50,784 & $35,556-77,378$ & $<.0001$ & .002 \\
\hline Short-term facility & 101,707 & $75,248-14,9040$ & & 80,743 & $45,402-127,557$ & & .04 \\
\hline Long-term facility & 92,452 & $67,677-132,809$ & & 106,184 & $70,271-155,959$ & & $<.0001$ \\
\hline Dead & 55,505 & $29,774-60,112$ & & 55,972 & $37,327-87,751$ & & .53 \\
\hline \multicolumn{8}{|c|}{ Age $\geq 65$ by discharge status } \\
\hline Home & 62,947 & $44,160-113,492$ & .0009 & 55,244 & $36,182-72,489$ & $<.0001$ & .03 \\
\hline Short-term facility & 101,330 & $73,270-163,007$ & & 92,509 & $61,951-123,834$ & & .46 \\
\hline Long-term facility & 99,571 & $75,600-134,762$ & & 92,603 & $58,976-137,171$ & & .28 \\
\hline Dead & 79,513 & $38,736-108,795$ & & 59,794 & $39,648-90,032$ & & .02 \\
\hline
\end{tabular}

Note:- - indicates no comparison performed.

tions were reimbursed through Medicare. Medicare reimbursement for hospitalization is bundled by means of DRGs. DRGs included in our comparison were MS-DRG 20 (intracranial vascular procedures with hemorrhage with major comorbidities and complications), 21 (intracranial vascular procedures with hemorrhage with comorbidities and complications), and 22 (intracranial vascular procedures with hemorrhage without comorbidities and complications).

\section{Statistical Analysis}

We used the nonparametric Wilcoxon rank sum test to determine statistical significance. In this study, we present medians and IQRs rather than means and SDs because the data were not normally distributed. ${ }^{2}$ Data on mean and SD costs are provided in the supplement. "Discharge Weights," provided by the NIS, were applied to all analyses. All statistical analyses were performed by using the SAS-based statistical software JMP (www.jmp.com).

\section{Results}

\section{Discharge Data}

Cost data from 2008 were available for a total of 10,422 discharges. Of these discharges, $4553(43.7 \%)$ were patients who underwent clipping and 5870 (56.3\%) underwent coiling. Eight hundred forty-three (18.5\%) discharges were for clipped patients, and 1562 discharges were for $(26.6 \%)$ coiled patients 65 years of age or older. Of discharges for clipped patients, 2575 (56.5\%) were discharged home, 1450 (31.9\%) were discharged to long-term facilities, and 528 (11.6\%) had in-hospital mortality. Of discharges for coiled patients, 3012 (51.3\%) were discharged home, 1989 (33.9\%) were discharged to long-term facilities, and $869(14.8 \%)$ had in-hospital mortality.

\section{Hospitalization Costs in 2008}

The median cost of hospitalization for all discharges of clipped patients was $\$ 69,353$ (IQR \$46,377-\$105,049); the median cost of hospitalization for discharges of coiled patients was $\$ 66,290$ (IQR, \$42,214-\$110,302) $(P=.45)$. Both age and discharge status had significant association with costs in clipped and coiled patients. The median cost of discharges for patients younger than 65 years of age undergoing clipping was significantly lower than that for patients 65 years of age or older ( $\$ 65,751$ versus $\$ 90,745$ respectively, $P<.0001$ ). The same was true for discharges of coiled patients because the discharges of patients younger than 65 years of age had a median cost of $\$ 64,252$ versus discharges for those patients 65 years of age or older who had a median cost of $\$ 74,918(P<$ $.01)$. There was a strong association between discharge status and costs in both age groups because in general, patients discharged home incurred lower costs than those discharged to short-term and long-term facilities. Hospitalization cost data are summarized in the Table. Data on mean costs are provided in the On-line Table.

\section{Medicare Population: Costs Compared with \\ Reimbursement}

For 2008, the average Medicare payment for craniotomy and endovascular intracranial procedures without complication (MS-DRG 22) was $\$ 30,380$. Medicare discharges with DRG 22 , for patients undergoing clipping, had a median cost of $\$ 59,799$ in 2008 . Those undergoing coiling had a median cost of $\$ 36,543$. Reimbursement for discharges of patients with complications or comorbidities (MS-DRG 21) was $\$ 36,304$. The median cost for hospitalization of clipped patients with MS-DRG 21 was $\$ 79,916$, and for coiled patients, $\$ 56,910$. 


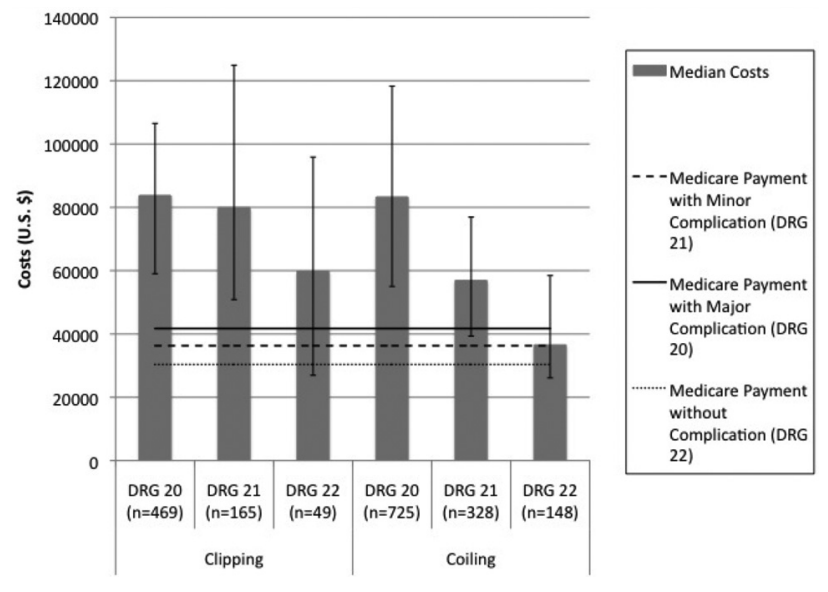

Fig 1. Costs in 2008 compared with 2008 Medicare payments.

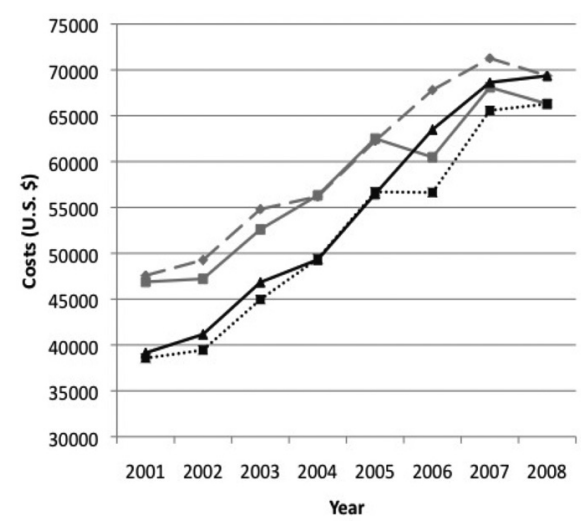

Fig 2. Costs for 2001-2008 for clipping and coiling of ruptured aneurysms.

Reimbursement for MS-DRG 20 (major complications or comorbidities) was $\$ 41,748$, with patients undergoing clipping incurring a median cost of $\$ 83,737$ and those undergoing coiling incurring a median cost of $\$ 83,277$. Hospitalization costs and Medicare payments are compared in Fig 1.

\section{Cost Trends with Time}

Trends in costs for treatment of ruptured aneurysms are summarized in Fig 2.

Between 2001 and 2008, the inflation adjusted cost (US \$ 2008) for hospitalization for treatment of a ruptured aneurysm with surgical clipping increased from $\$ 47,580$ (IQR = $\$ 31,725-\$ 72,808)$ to $\$ 69,353$ (IQR $=\$ 46,377-\$ 105,049)$, which amounts to a $5.7 \%$ annual inflation rate. During this same period, the cost associated with coiling of ruptured aneurysms increased from $\$ 46,881(\mathrm{IQR}=\$ 26,928-\$ 76,764)$ to $\$ 66,290$ ( IQR $=\$ 42,214-\$ 110,272)$, which amounts to a $5.2 \%$ annual inflation rate.

\section{Discussion}

Overall, we found that costs for treatment of ruptured cerebral aneurysms are substantially higher than the average Medicare payments for all patients regardless of degree of complications and comorbidities. Twenty-three percent of patients undergoing clipping or coiling for ruptured aneurysm in the United States were 65 years of age or older, so Medicare patients represent a significant minority of these cases.
Hoh et $\mathrm{al}^{3}$ reported on the cost of cerebral aneurysm treatment in a study of the NIS for 2002-2006, finding that clipping was associated with significantly longer lengths of stay and significantly higher total hospital charges than coiling for patients with ruptured cerebral aneurysms. We sought to further delineate the reasons for cost differentials in ruptured aneurysms by stratifying patients by age and outcome and by evaluating the trend in cost with time. We found that the charges were not significantly different for clipping and coiling in patients younger than 65 years of age, but clipping was associated with significantly higher costs than coiling in patients 65 years of age or older. Hospitalization costs for both coiling and clipping increased from 2001 to 2008 , but the costs for the 2 procedures remained similar to each other during that period (Fig 2). A single center study from the University of Florida found that coiling tended to lead to higher hospital charges than clipping of ruptured aneurysms, ${ }^{4}$ demonstrating that a single center might have costs that deviate from the norms of the NIS data base.

The NIS does not provide itemized cost information, which would allow determination of specific sources of the increase in cost with time. Because the costs of hospitalization are rising in a quite similar pattern, the major contributor may be general inflation of hospital costs rather than expenses specific to the procedures such as device costs. It is possible that hospitals in the United States could succeed in a national effort to reduce overall hospital expenses, which would almost certainly bring down the cost of care for patients with subarachnoid hemorrhage. A report from Pakistan found that from 2004 to 2007, the cost of clipping was $\$ 3127$, whereas the cost of coiling was higher at $\$ 5080$, largely due to higher procedure price and cost of consumables. ${ }^{5}$ It is difficult to directly compare costs of hospitalization between different countries because of wide differences in nearly all itemized costs due to fundamental economic differences.

The hospital costs for patients in the NIS treated for ruptured aneurysms was highly dependent on the presence of morbidity or mortality. For patients who had morbidity and mortality, the cost of critical care overwhelmed the differences in periprocedural charges for coiling and clipping. It is not possible to separate complications of the aneurysm treatment from the complications of the initial subarachnoid hemorrhage by using the NIS.

Later charges for follow-up imaging surveillance for aneurysm recurrence are likely to lead to an increase in costs associated with coiling. Also, a higher recurrence rate of coiled aneurysms potentially leading to a need for additional treatment with hospitalization will add further costs to a significant fraction of coiled patients. The International Subarachnoid Aneurysm Trial showed relatively little difference in rerupture rates between coiled and clipped aneurysms, ${ }^{6}$ so differences in hospitalization for subsequent rupture of coiled versus clipped aneurysms are not likely to add to differences in hospital charges. A cost-effectiveness study looking at costs of clipping versus coiling for an entire year, including angiographic follow-up and retreatment, found that the cost of coiling was higher but nonetheless cost-effective. ${ }^{2}$

Large aneurysms and posterior circulation aneurysms might be associated with higher charges for either clipping, coiling, or both. ${ }^{3}$ The NIS provides no information on aneu- 
rysm size or location, so we cannot evaluate these parameters. Another potential limitation of this study is the occurrence of coding errors, which are present in any large administrative data base. ${ }^{7}$ However, errors in databases of this type are expected to be randomly distributed because recording is done by clerical and coding staff remote from the study questions. Furthermore, random coding errors are probably diluted in such a large sample and thus are not likely to significantly affect overall conclusions. Another limitation is that the hospital charge data are given only as a total, so we are unable to evaluate individual components of those charges. Payments associated with MS-DRG 20, 21, or 22 for patients who did not have a cerebral aneurysm coiled or clipped were not included in this analysis, which would include patients undergoing treatment for brain arteriovenous malformations.

\section{Conclusions}

Hospitalization costs for patients undergoing clipping and coiling of ruptured cerebral aneurysms are substantially higher than corresponding Medicare payments. Effort to decrease hospitalization costs and/or increase payments will be necessary to make the care of patients with ruptured aneurysms economically viable for hospitals in the long term.

Disclosures: Harry J. Cloft-UNRELATED: Research Support. Mindframe, Cordis Endovascular, Grants/Grants Pending: Cordis, Medtronic. David Kallmes_UNRELATED: Research Support. ev3, MicroVention, Chestnut, Micrus Endovascular, Grants/Grants Pending: ev3,*
Penumbra, ${ }^{*}$ NFocus, ${ }^{*}$ Micrus, ${ }^{*}$ MicroVention, ${ }^{*}$ Cook ${ }^{*}$ Arthrocare, ${ }^{*}$ Payment for Development of Educational Presentations: ev3, ${ }^{*}$ CareFusion, ${ }^{*}$ Travel/Accommodations/Meeting Expenses Unrelated to Activities Listed: MicroVention. * Giuseppe Lanzino-UNRELATED. Educational Grants: ev3, ${ }^{*}$ Synthes, ${ }^{*}$ Advisory Board: Actelion Pharmaceuticals. *Payment made to the institution.

\section{References}

1. Qureshi AI, Vazquez G,Tariq N, et al. Impact of International Subarachnoid Aneurysm Trial results on treatment of ruptured intracranial aneurysms in the United States. J Neurosurg 2011;114:834-41

2. Hoh BL, Chi YY, Lawson MF, et al. Length of stay and total hospital charges of clipping versus coiling for ruptured and unruptured adult cerebral aneurysms in the Nationwide Inpatient Sample database 2002 to 2006. Stroke 2010;41:337-42

3. Hoh BL, Chi YY, Dermott MA, et al. The effect of coiling versus clipping of ruptured and unruptured cerebral aneurysms on length of stay, hospital cost, hospital reimbursement, and surgeon reimbursement at the University of Florida. Neurosurgery 2009;64:614-19

4. Zubair Tahir M, Enam SA, Pervez Ali R, et al. Cost-effectiveness of clipping vs coiling of intracranial aneurysms after subarachnoid hemorrhage in a developing country: a prospective country. Surg Neurol 2009;72:355-60, discussion 360-61. Epub 2009 Jul 17

5. Molyneux AJ, Kerr RS, Birks J, et al, for the ISAT Collaborators. Risk of recurrent subarachnoid haemorrhage, death, or dependence and standardised mortality ratios after clipping or coiling of an intracranial aneurysm in the International Subarachnoid Aneurysm Trial (ISAT): long-term follow-up. Lancet Neurol 2009;8:427-33. Epub 2009 Mar 28

6. Maud A, Lakshminarayan K, Suri MF, et al. Cost-effectiveness analysis of endovascular versus neurosurgical treatment for ruptured intracranial aneurysms in the United States. J Neurosurg 2009;110:880-86

7. Woodworth GF, Baird CJ, Garces-Ambrossi G, et al. Inaccuracy of the administrative database: comparative analysis of two databases for the diagnosis and treatment of intracranial aneurysms. Neurosurgery 2009;65:251-56 